Original

\title{
Mecanismo fisiopatológico de los sofocos-sudores inducidos por deprivación androgénica en el carcinoma prostático
}

\author{
Santiago Vilar Gónzalez*, Francesc Montañá Puig**, Mª Victoria Villas Sánchez*, \\ $\mathrm{M}^{\mathrm{a}}$ del Mar Sevillano Capellán*, Manuel Aguayo Martos*, Sebastiá Sabater Martí* \\ ${ }^{*}$ Complejo Hospitalario Universitario de Albacete (CHUA). ${ }^{* *}$ Angelini-Farmacéutica. Barcelona, España
}

\begin{abstract}
Resumen
Los sofocos-sudores son problemas frecuentes durante el tratamiento con deprivación androgénica en pacientes afectos de carcinoma prostático. Estos efectos secundarios contribuyen en gran medida al deterioro de la calidad de vida.

Se han postulado diferentes hipótesis sobre los mecanismos fisiopatogénicos implicados, todos ellos de una alta complejidad debida básicamente a su multicausalidad.

El objetivo de esta revisión es aportar una visión meditada y actualizada de su génesis.

La retroalimentación negativa de las hormonas sexuales plasmáticas sobre la secreción hipotalámica de noradrenalina y serotonina serían la causa principal.

Asimismo la acción directa de estos mecanismos sobre el centro hipotalámico productor de LH-RH cercano al centro termorregulador, junto al acortamiento del intervalo termoneutral también estarían implicados.

La mejor comprensión de su mecanismo de producción nos puede hacer enfocar mas correctamente su tratamiento.
\end{abstract}

Palabras clave: Carcinoma prostático. Sofocos. Deprivación hormonal.

\section{Physiopathological mechanism of hot flushes-perspiration induced by androgen deprivation in prostate carcinoma}

\section{Abstract}

Hot flushes and perspiration are common problems during androgen deprivation therapy for prostate carcinoma, and largely contribute to worsen patient quality of life.

Different hypotheses have been proposed to explain the underlying physiopathological mechanisms, though all are very complex, basically because of the multiple causal factors involved.

The present review offers a pondered and updated perspective of the origin of hot flushes-perspiration in such patients.

Negative feedback of the plasma sexual hormones upon the hypothalamic secretion of noradrenalin and serotonin appears to be the main cause. Likewise, the direct action of such mechanisms upon the LH-RH producing hypothalamic center located close to the thermoregulatory center, together with shortening of the thermoneutral interval, would also play a role.

Improved understanding of the causal mechanism may help improve the treatment of such symptoms.

Keywords: Prostate carcinoma. Hot flushes. Hormone deprivation.

$\mathrm{E}$ cáncer de próstata es el segundo tumor en importancia en el varón, con aproximadamente 680.000 nuevos casos al año en el mundo y representa el 11,7\% de todos los tumores masculinos. En España se diagnostican unos 13.300 casos anuales, lo que representa el 13,6\% de los tumores entre los hombres. La incidencia en España, comparada con el resto de países desarrollados, se puede considerar baja (tasa ajustada mundial en 2002: 36 nuevos casos/100.000 habitantes/año) ${ }^{1,2}$.

La terapia de deprivación androgénica que se asocia al tratamiento de determinados cánceres de próstata, sea con análogos de la hormona liberadora de gonadotrofinas (LH-RH), antiandrogénicos orales y/o orquidectomía bilateral, inducen a una variada sintomatología hormonodependiente de entre la cual nos referiremos a los sofocos-sudores ${ }^{3}$. A partir de ahora nos referiremos a ellos únicamente como sofocos.

En 1896, Cabot estudió los efectos de la castración en el tratamiento del carcinoma prostático describiéndolos por primera vez ${ }^{4}$. Medio siglo después, en 1941 Huggins y Hodges, demostraron la correlación entre cáncer de próstata y andrógenos ${ }^{5}$. También describieron las crisis de sofocos en 9 de 21 pacientes castrados, tras 2 a 3 semanas de la cirugía. 
Los sofocos se definen como una sensación subjetiva de calor, principalmente en la parte superior del torso, seguido de una excesiva transpiración. El síntoma primario es la sensación subjetiva de calor $^{6,7}$ que puede durar varios minutos (de 4 a 10) pudiendo ir acompañado de otros síntomas como ansiedad, irritabilidad, palpitaciones, rubefacción, pánico y sensación de pérdida de control, con gran repercusión física y emocional ${ }^{7-9}$. La temperatura corporal sube una media de $0,9^{\circ} \mathrm{C}$ entre 7 a 20 minutos antes del sofoco, con el consiguiente aumento de emisión de energía y del coeficiente respiratorio $^{10}$.

Existe una vasodilatación periférica generalizada ${ }^{11,12}$ que ocurre típicamente en los primeros segundos del inicio del cuadro ${ }^{13}$. Asimismo se incrementa la transpiración y la conductancia eléctrica, existiendo una gran correlación entre ambas mediciones $^{12}$. Se presenta un incremento de $2-\mathrm{mS}$ durante 30 segundos en la conductancia cutánea durante la clínica de sofocos ${ }^{14-16}$. La medición de la conductancia cutánea podría ser de interés para la monitorización de la clínica de sofocos de manera independiente a la percepción del paciente, al ser factible su medición de manera ambulatoria mediante un mecanismo semejante al Holter. De todas maneras existe una gran concordancia entre estos registros y los obtenidos mediante cuestionarios diarios de recogida de información ${ }^{14-16}$.

\section{DESARROLLO}

El motivo del presente trabajo de revisión es analizar la fisiopatogénesis de dichos sofocos. Con tal finalidad y tras una búsqueda bibliográfica inicial en Medline, que comprendió los últimos 5 años, se han analizado los resultados y solicitado aquellos trabajos que han parecido de interés. Asímismo, las referencias bibliográficas de sus autores se han tomado como fuente para poder disponer de un mayor número de artículos. Todas las publicaciones referenciadas se han analizado a partir de los trabajos originales.

Desde los trabajos pioneros de Huggins y Hodges $^{5}$, la manipulación hormonal para el manejo del cáncer de próstata ha ido adquiriendo un mayor protagonismo. El tratamiento consiste en deprivar de andrógenos a las células cancerosas. Hasta hace algunos años la práctica habitual era la orquidectomía bilateral que ha sido paulatinamente sustituida por la administración de análogos LH-RH.
Tras la orquidectomía bilateral, alrededor del $50 \%$ de los pacientes presentaban sofocos durante algunos meses ${ }^{17-20}$.

Aunque algunos estudios han fallado a la hora de mostrar una correlación entre los niveles preoperatorios de testosterona y los sofocos ${ }^{17-21}$, otros han reportado mayor incidencia en personas jóvenes con mayores niveles de testosterona ${ }^{22}$.

Con análogos LH-RH la incidencia de sofocos es entorno al 60-75\% ${ }^{23-26}$. Esta mayor incidencia con respecto a la orquidectomía no es bien conocida. En paralelo con la orquidectomía, los sofocos se presentan durante varios meses pudiendo persistir aunque en menor intensidad y frecuencia durante años.

Es bien conocido que los sofocos son un problema clínico significativo en los pacientes con carcinoma prostático y deprivación androgénica ${ }^{17,27,28}$. $\mathrm{Su}$ significación ha quedado evidenciada en los estudios sobre calidad de vida llevados a cabo en pacientes tratados con dicha terapia ${ }^{29}$.

\section{RESULTADO: FISIOPATOGÉNESIS}

Las crisis de sofocos ocurren frecuentemente en asociación con la menopausia y el fallo ovárico, de ahí que se pueda asumir que sean motivados por los bajos niveles de hormonas sexuales. La teoría de la deprivación hormonal en el hombre con carcinoma prostático se muestra atractiva y se sustenta en la aparición de las crisis del sofoco tras la ooforectomía ${ }^{30}$ y tras la castración, tanto médica ${ }^{26,31,32}$ como quirúrgica ${ }^{33}$. Asimismo, esta teoría se ve favorecida por la respuesta a la terapia hormonal sustitutiva o tras su abandono ${ }^{34,35}$. En su contra, cabe señalar la ausencia de esta clínica en el hipogonadismo congénito (sindromes de Turner y Kallman) y en la prepubertad $^{36}$.

Todo ello sugiere, que no es la concentración absoluta de hormonas sexuales en plasma sino su reducción dinámica, su deprivación abrupta, lo que genera la clínica. Esta es, actualmente, la teoría más aceptada ${ }^{36}$. En el siguiente desarrollo se pueden extrapolar los hallazgos en los estudios sobre los estrógenos al efecto ejercido a nivel central de los andrógenos.

En 1976, Alksel $^{37}$ cuestionó la teoría de la deprivación, sugiriendo que los sofocos eran el resultado de la elevación de los niveles de gonadotrofinas; postulando una causa pituitaria opuesta a la gonadal. Estudios en mujeres premenopáusicas que correla- 
cionan los niveles plasmáticos de hormona luteinizante (LH) y folículo estimulante (FSH) con los sofocos apoyan dicha teoría ${ }^{38}$. Los grupos de Casper $^{39}$ y Tataryn $^{3}$ muestran una sincronía entre la ocurrencia de picos de LH y los sofocos yendo un paso más allá y proponiendo la teoría pulsátil ${ }^{36}$. En su contra, indicar, que no todas las elevaciones de LH se acompañan de sofocos aunque todos los sofocos sí van acompañados de un pico de LH.

En contraste con todo esto no se observa correlación entre los niveles de gonadotrofinas con la clínica de sofocos en las mujeres posmenopáusicas $^{40,41}$. Señalar también, la ausencia de sofocos en el sindrome de Klinefelter (con altos niveles de gonadotrofinas) y en tratamientos que inducen niveles elevados de gonadotrofinas, como son los protocolos utilizados en la inducción a la ovulación. Sin embargo, sí pueden existir sofocos tras hipofisectomías y durante la terapia con agonistas LH-RH para el cáncer de próstata.

Todo ello ha orientado a investigadores hacia un probable origen hipotalámico de la fisiopatología de los sofocos ${ }^{36}$

Similitudes entre la clínica del sofoco y síntomas neurovegetativos adrenérgicos sugieren que las catecolaminas pudieran estar implicadas ${ }^{7}$. Casper ${ }^{39}$ falló a la hora de mostrar la correlación entre los sofocos y los niveles periféricos de catecolaminas, concluyendo que las vías implicadas debían ser centrales.

Por otro lado existen evidencias de que la noradrenalina (NA) es el neurotransmisor hipotalámico responsable de los sofocos. Beckman ${ }^{42}$ demostró que en animales de experimentación la administración intrahipotalámica de NA afectaba a la termorregulación ${ }^{7}$. Respalda dicha hipótesis las elevadas concentraciones periféricas de MHPG (3-metoxi-4hidroxi-fenilglicol) en las crisis de sofocos, el principal metabolito de la NA procedente del cerebro, mientras que no se observan alteraciones de la concentración de VMA (Ácido vanilmandélico), metabolito de la NA de producción periférica ${ }^{7,43}$.

Todo ello se ve refrendado tras la administración de yohimbina ( $\alpha_{2}$-antagonista) que eleva las concentraciones hipotalámicas de NA generando sofocos en mujeres ya sintomáticas, mientras que estos ceden tras la administración de clonidina ( $\alpha_{2}$-agonista $)^{44}$. La elevada activación neurovegetativa simpática, vehiculizada por el receptor $\alpha_{2}$-adrenérgico juega el papel principal en la activación del sofoco. Así, ya que los estrógenos modulan a estos receptores, es posible que la abrupta deprivación estrogénica este implicada en la fisiopatogénesis de los sofocos por esta vía ${ }^{45}$. Se ha observado que la exposición a altas temperaturas o los alimentos calientes pueden disminuir el número de estos receptores, liberándose más NA y produciéndose la clínica de $\operatorname{sofocos}^{36}$.

Por otro lado, el centro termorregulador hipotalámico se haya anatómicamente muy cercano al centro productor de LH-RH. Incrementos de la NA hipotalámica por la deprivación hormonal estimularían por tanto a las neuronas productoras de LH-RH (retroalimentación o Feedback) y por proximidad al centro termorregulador, activando los mecanismos de pérdida de calor ${ }^{46-48}$. Estos incluirían la vasodilatación cutánea y la transpiración profusa. Asimismo, se ha evidenciado que elevados niveles de NA disminuyen el umbral de los sofocos-sudores en mujeres posmenopáusicas sintomáticas provocando su aparición ${ }^{7,49}$.

La asociación entre los esteroides sexuales y las catecolaminas hipotalámicas no es bien conocida. Se ha propuesto la hipótesis de los catecolestrógenos (2hidroxi-estrógeno $)^{50,51}$. Ellos son los metabolitos más comunes de los estrógenos, presentando similar estructura que las catecolaminas. La concentración de catecolestrógenos en el hipotálamo es 10 veces superior a la de estrógenos ${ }^{21}$. Los catecolestrógenos actúan sobre la catecolmetiltransferasa y la tirosinhidroxilasa las cuales a su vez operan sobre la sintesis y degradación de las catecolaminas, disminuyendo los niveles de NA. Por lo tanto, concentraciones bajas de catecolestrógenos en el hipotálamo darán lugar a un incremento de las concentraciones de NA y con ello la aparición clínica de sofocos.

Por otro lado, también los opioides están implicados en la patogénesis de los sofocos ${ }^{45}$. Stubbs et al. ${ }^{52}$ generaron sofocos tras administrar opioides en pacientes sanos. La naloxona, un antagonista opioide, genera sintomas vasomotores al ser administrada en animales opiodependientes ${ }^{53,54}$. Los esteroides sexuales incrementan las $\beta$-endorfinas, opioides endógenos hipotalámicos 55,56 . Las $\beta$-endorfinas aumentan la síntesis de catecolestrógenos los cuales inhiben la sintesis hipotalámica de NA ${ }^{14,47,55-58}$ (Fig. 1).

Una deprivación abrupta de hormonas sexuales dará lugar, por tanto, a una disminución de los niveles de endorfinas y en la pérdida de la retroalimentación negativa $6,47,56$. 


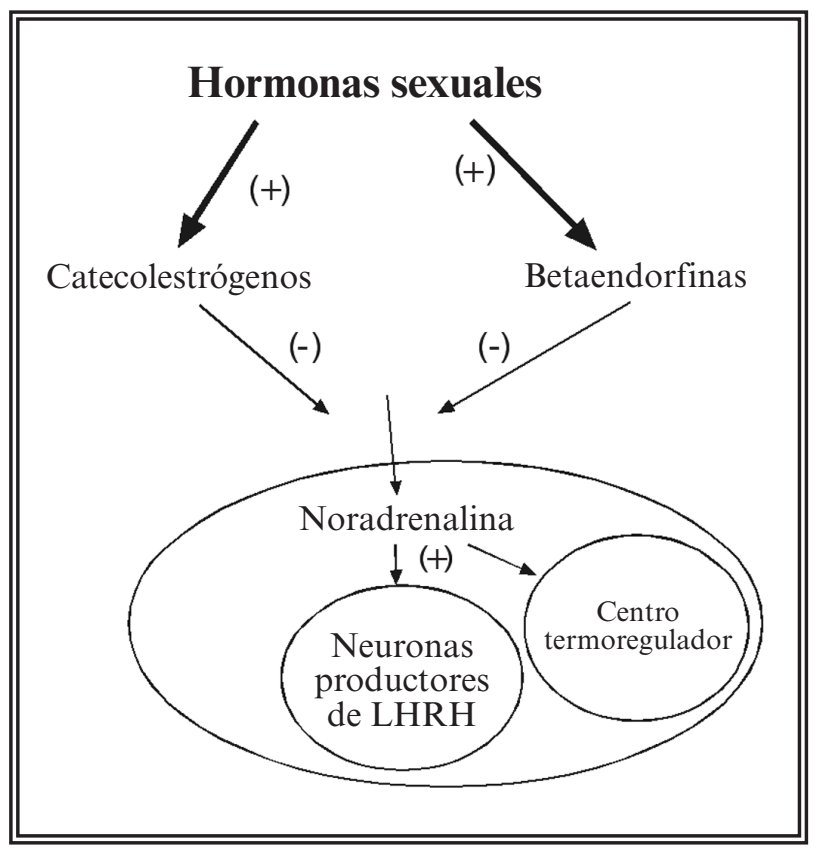

FIGURA 1. Feedback negativo de las hormonas sexuales sobre la noradrenalina hipotalámica.

Como resultado de la disminución de los niveles hipotalámicos de catecolestrógenos y betaendorfinas, los niveles hipotalámicos de NA se verán incrementados. Esta va a estimular a las neuronas productoras de LH-RH provocando su secreción y por cercanía, actuará sobre el centro termorregulador acortando su intervalo de respuesta, rebajando sus umbrales de tolerancia a la temperatura y generando la clínica de sofocos ${ }^{14,47,59}$.

En el centro regulador existe un intervalo ("ventana”) para el control térmico corporal, limitado por un umbral inferior que pone en marcha los escalofríos como respuesta para provocar un incremento de la temperatura; y un umbral superior que como hemos indicado desencadena la pérdida de calor por medio de la vasodilatación cutánea y la sudación profusa al activar el sistema neurovegetativo ${ }^{14,48,60,61}$ (Fig. 2).

En los pacientes afectos de sofocos, el intervalo termoneutral se ve muy acortado y pequeños cambios de la temperatura tiene efectos sobre el centro termoregulador ${ }^{10,14,43,49}$. El aumento de la temperatura corporal a raíz de un aumento de la temperatura ambiente o la ingesta de alimentos demasiado calientes, provoca que se sobre-

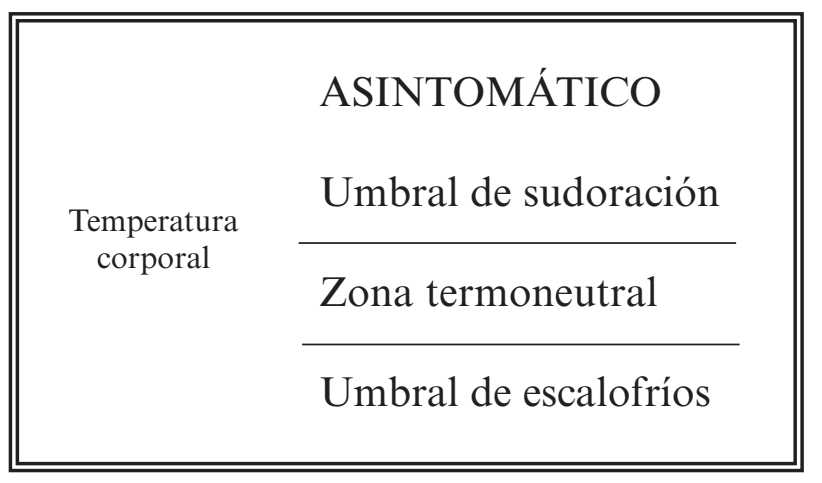

FIGURA 2. Intervalo de control térmico corporal.

pase el umbral superior del intervalo termoneutral y se disparen los mecanismos disipadores de calor (vasodilatación, sudación) 14,61,62 (Fig. 3).

Sustancias como la noradrenalina, al aumentar su concentración en el hipotálamo generan este acortamiento del intervalo mientras que sustancias como la serotonina $7,14,43,55,56,63,64$ y la dopamina ${ }^{65}$ tienen el efecto contrario. Se ha llegado a la conclusión de que el intervalo termoneutral es de $0,8{ }^{\circ} \mathrm{C}$ en los pacientes asintomáticos y de $0,0{ }^{\circ} \mathrm{C}$ en los sintomáticos ${ }^{14}$ (Fig. 4).

Los niveles de serotonina (5-hidroxitriptamina o 5-HT) en mujeres posmenopáusicas están disminuidos, normalizándose con las terapias sustitutivas. Todo ello sugiere que la deprivación abrupta de hormonas sexuales da lugar a una reducción en la circulación de serotonina, con el consiguiente aumento de sus receptores $5-\mathrm{HT}_{2 \mathrm{~A}}$ hipotalámicos ${ }^{7,56}$. Podrían estar estos receptores muy implicados en la patogénesis de los sofocos ${ }^{7}$ (Fig. 5).

\section{CONCLUSIÓN}

La deprivación abrupta de andrógenos inducidos tanto por la orquidectomía como sobre todo por el bloqueo hormonal mediante análogos LH-RH, genera un aumento de la concentración hipotalámica de NA

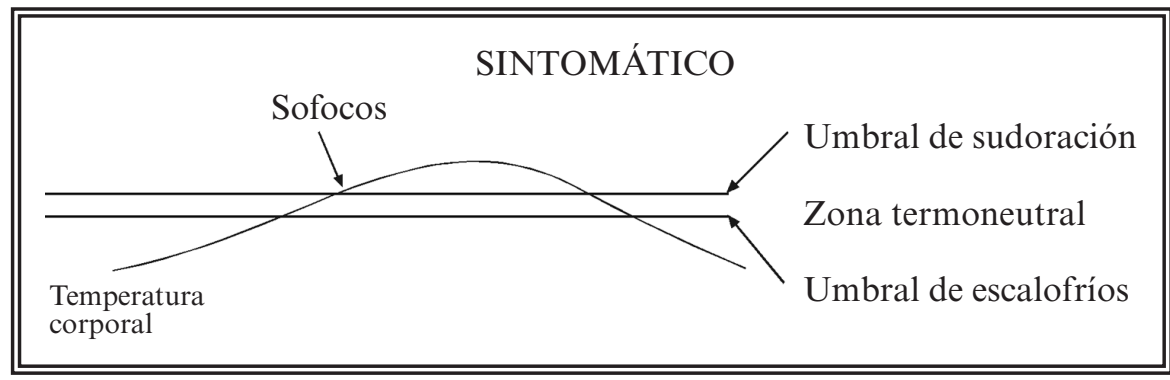

FIGURA 3. Un intervalo termorregulador (zona termoneutral) acortado provoca una gran sensibilidad del centro termorregulador. 


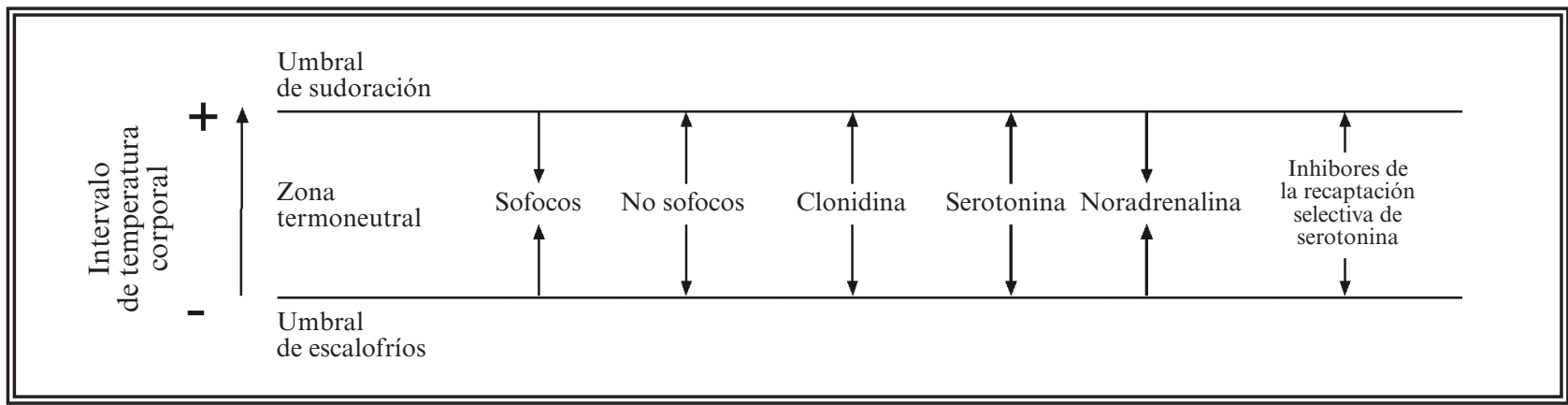

FIGURA 4. La zona termoneutral varia según sean los pacientes sintomáticos $\left(0,0^{\circ} \mathrm{C}\right)$ o no $\left(0,8^{\circ}\right)$.

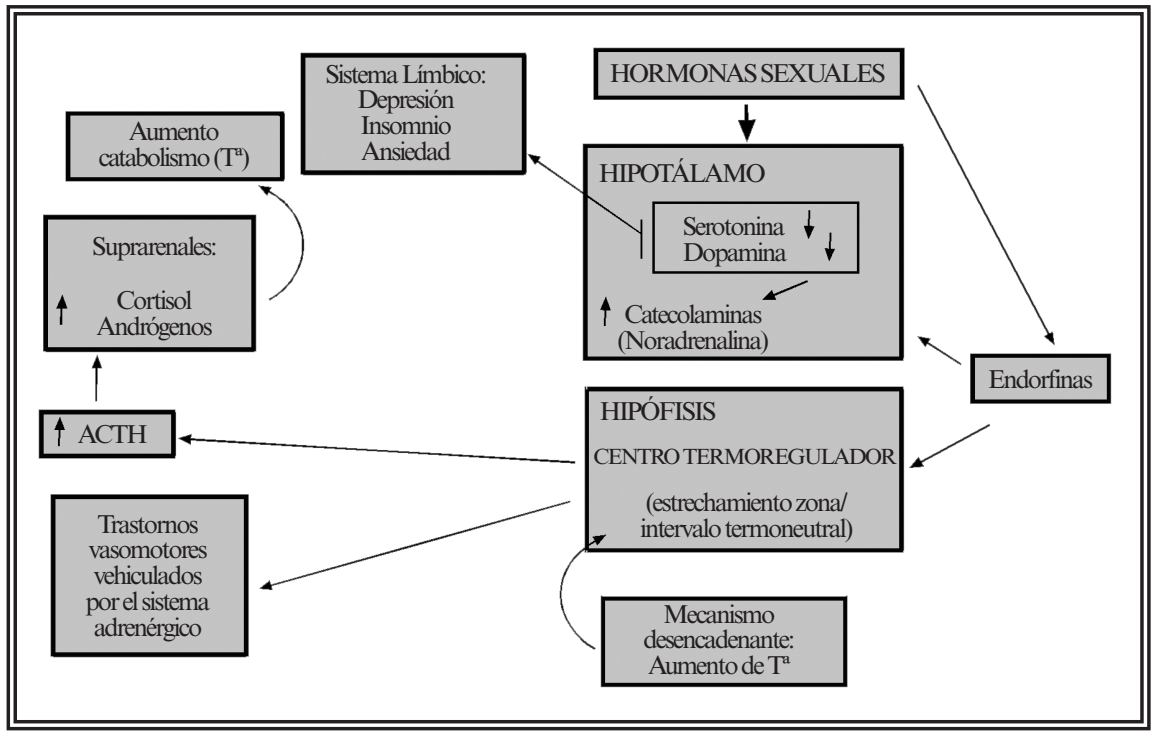

FIGURA 5. En la fisiopatogénesis de los sofocos es importante el feedback negativo de las hormonas sexuales plasmáticas sobre la secreción hipotalámica de noradrenalina y serotonina.

por un mecanismo de retroalimentación, de cara a intentar normalizar de nuevo los niveles de hormonas sexuales.

Este estímulo a nivel del centro productor del LH-RH genera que por cercanía también se estimule el centro termorregulador desencadenándose el mecanismo de los sofocos-sudores.

Las vías implicadas en el aumento de los niveles de NA son la menor presencia de betaendorfinas y catecolestrógenos (o sus homólogos androgénicos), que no ejercerán su papel inhibiendo la síntesis de NA, así como la menor presencia de los receptores $\alpha_{2}$-adrenérgicos, los cuales también se verán disminuidos por las altas temperaturas y los alimentos calientes aumentando la producción de NA y desencadenándose la clínica de sofocos.

Por otro lado, la menor concentración de hormonas sexuales también tendrá su reflejo en una menor concentración de serotonina y un aumento de sus receptores 5$\mathrm{HT}_{2 \mathrm{~A}}$ a nivel hipotalámico. Estos receptores pueden jugar un papel clave en la génesis y por extensión en el enfoque terapéutico de esta sintomatología.

Al mismo tiempo la mayor presencia de NA y menor de serotonina generan un acortamiento en el intervalo termoneutral, siendo estos pacientes más susceptibles a pequeños cambios de la temperatura que desencadenarían la clínica.

Por todo ello y para concluir señalar en la fisiopatogénesis de los sofocos lo importante que es comprender la retroalimentación negativa de las hormonas sexuales plasmáticas en la secreción hipotalámica de noradrenalina y su balanceo con la serotonina.

La mejor comprensión del mecanismo generador de los sofocos-sudores nos puede hacer enfocar mejor el abordaje terapéutico de esta sintomatología.

\section{REFERENCIAS}

1. López-Abente G. La situación del Cáncer en España. Ministerio de Sanidad y Consumo. Madrid 2005.

2. Vera Donoso CD. El tratamiento del cáncer de próstata: Presente y futuro, realidades y posibilidades. Actas Urol Esp. 2007 31(6):575-579.

3. Tataryn IV, Meldrum DR, Lu KH, Frumar AM, Judd HC. LH, FSH and skin temperature during the menopausal hot flush. J Clin Endocrinol Metab. 1979;49(1):152-154.

4. Cabot AT. The question of castration for enlarged prostate. Ann Surg. 1896;24(3):265-309.

5. Huggins C, Hodges GV. Studies on prostate cancer. I. The effect of castration, estrogen and androgen injections on serum phosphatases in Metastatic carcinoma of the prostate. CA Cancer $J$ 
Clin. 1972;22(4):232-240.

6. Loprinzi Cl, Barton DL, Carpenter LA et al. Pilot evaluation of paroxetine for treating hot flashes in men. Mayo Clin Proc. 2004;79(10):1247-51.

7. Albertazzi P. Nonadrenergic and serotonergic modulation to treat vasomotor symptoms. J Br Menopause Soc. 2006;12(1):7-11.

8. Suvanto-Luukkonen E, Koivunen R, Sundström H, Bloigu R, Karjalainen E, Häivä-Mällinen L, Tapanainen JS. Citalopram and fluoxetine in the treatment of postmenopausal symptoms: a prospective, randomized, 9-month, placebo-controlled, double-blind study. Menopause, 2005;12(1):18-26.

9. Kronenberg Fl. Hot flashes: phenomenology, quality of life and search for treatment options. Exp Gerontol. 1994;29(3-4):319 336.

10. Carpenter JS, Gilchrist JM, Chen K, Gautam S, Freedman RR. Hot flashes, core body temperature and metabolic parameters in breast cancer survivors. Menopause. 2004;11(4):375-381.

11. Jessen AB, Toubro S, Astrup A. Effects of chewing gum containing nicotine and caffeine on energy expenditure and substrate utilization in men. Am J Clin Nutr. 2002; 77(6):1442-1447.

12. Molnar GW. Body temperature during menopausal hot flashes. J Appl Physiol, 1975;38(3):499-503.

13. Kronenberg F. Menopausal hot flashes: thermoregulatory, cardiovascular and circulating catecholamine and LH changes. Maturitas. 1984;6(1):31-43

14. Freedman RR Hot flashes: behavioral treatments, mechanims, and relation to sleep. Am J Med 2005;118(12 Suppl 2):124-130.

15. Laboratory and ambulatory monitoring of menopausal hot flashes. Psychophysiology. 1989; 26 (5): 573-579.

16. Carpenter JS, Andrykowski MA, Freedman RR, Munn R. Feasibility and psychometrics of an ambulatory hot flash monitoring device. Menopause, 1999;6(3):209-215.

17. Charig CR, Rundle JS. Flushing. Long-term side effects of orchidectomy in treatment of prostatic cancer. Urology. 1989;33(3): 175-178.

18. Varenhorst E, Frödin T, Alund G. Climacteric flushing in a man. Br Med J (ClinRes Ed). 1983; 287(6395):838-839.

19. Maatman TJ, Gupta MK, Montie JE. Effectivenes of castration vs intravenous estrogen in producing rapid endocrine control of metastatic cancer of prostate. J Urol. 1985;133 (4):620-621.

20. Hammond MG, Hatley L, Talbert LM. Hammond MG et al. A double blind study to evaluate the effect of methyldopa on menopausal vasomotor flushes. J Clin Endocrinol Metab. 1984; 58 (6): 1158-1160.

21. Smith JA. Management of hot flushes due to endocrine therapy for prostate cancer. Oncology. 1996;10(9):1319-1322.

22. Buchholz NP, Matarelli G. Hot flushes after orchidectomy in treatment of prostate cancer - a serious effect. Zeitschrift fur Gerontologie. 1994;27(5):334-336.

23. Leuprolide Study Group. Leuprolide vs diethylstilbestrol for metastatic prostate cancer. N Engl J Med. 1984;311(2):12811286.

24. Sarosdy MF, Schellhammer PF, Soloway MS et al. Endocrine effects, efficacy and tolerability of a $10,8 \mathrm{mg}$ depot formulation of goserelin acetate administered every 13 weeks to patients with advanced prostate cancer. BJU Int. 1999;83(7):801-806.

25. Parmar H, Edwards L, Phillips RH, Allen L, Lightman SL. Orchidectomy vs long acting D-Trip-6-LHRH in advanced prostate cancer. BJU Int. 1987;59(3):248-254

26. Burch PA. Prostate-specific antigen decline after withdrawal of low-dose megestrol acetate (letter). J Clin Oncol, 1999; 17(3): 1087-1088.

27. Quella SK, Loprinzi CL, Sloan J, et al. Pilot evaluation of venlafaxine for the treatment of hot flashes in men undergoing androgen ablation therapy for prostate cancer. Journal of Urology. 1999;162(1):98-102.
28. Nishiyama $\mathrm{T}$, Kanazawa $\mathrm{S}$, Watanabe $\mathrm{R}$, Terunuma M, Takahashi K. Influence of hot flashes on quality of life in patients with prostate cancer treated with androgen deprivation therapy. Int J Urol. 2004;11 (9): 35-741.

29. Loprinzi CL, Pisansky TM, Fonseca R, Sloan JA, Zahasky KM, Quella SK, Novotny PJ, Rummans TA, Dumesic DA, Perez EA. Pilot evaluation of venlafaxine hydrochloride for the therapy of hot flashes in cancer survivors. J Clin Oncol. 1998;16(7):2377-2381.

30. Chakravarti S, Collins WP, Forecast JD, Newton JR, Oram DH, Studd JW. Hormone profiles after the menopause. Br Med J. 1976;2(6039):784-787.

31. Shamash J, Davies A, Ansell W, et al. A phase II study investigating the re-induction of endocrine sensitivity following chemotherapy in androgen-independent prostate cancer. $\mathrm{Br} \mathrm{J}$ Cancer. 2008;98(1):22-24.

32. Holdaway IM, Ibbertson HK, Croxson MS, et al. Treatment of metastatic prostate cancer with depot LH-RH analogue Zoladex. Prostate. 1988;12 (2):119-127.

33. Béland G, Elhilali M, Fradet Y, et al. Total androgen blockade vs castration in metastatic cancer of the prostate. Hormonal Therapy of prostatic Disease: Basic and Clinical Aspects. 35. Proceeding of an International Symposium. Milan, Italy April 68. Medicom, 1987; 243A: 391-400.

34. Frödin T, Alund G, Varenhorst E. Measurement of skin bloodflow and water evaporation as a means of objectively assessing hot flushes after orchidectomy in patients with prostatic cancer. Prostate. 1985;7(2):203-208.

35. Coope J, Thomson JM, Poller L. Effects of "natural oestrogen" replacement therapy on menopausal symptoms and blood clotting. Br Med J. 1975;4(5989): 139-143.

36. Kouriefs C, Georgiou M, Ravi R. Hot flushes and prostate cancer: pathogenesis and treatment. BJU International. 2002;89 (4):379-383.

37. Aksel S, Schomberg DW, Tyrey L, Hammond CB. Vasomotor symptoms, serum estrogens, and gonadotropin levels in surgical menopause. Am J Obstet Gynecol. 1976;126(2):165-169.

38. Meldrum DR, Defazio JD, Erlik Y, et al. Pituitary hormones during the menopausal hot flash. Obstet Gynecol. 1984;64(6): 752-756.

39. Casper RF, Yen SS, Wilkes MM. Menopausal flushes: a neuroendocrine link with pulsatile luteinizing hormone secreation. Science. 1979;205(4408):823-825.

40. Linsell CR, Lightman SL. Postmenopausal flashes: studies of chronological organisation. Psychoneuroendocrinology. 1983;8 (4):435-440.

41. Daw E. Luteinizing hormone (LH) changes in female undergoing artificial menopause. Curr Med Res Opin. 1974;2(5):256-259.

42. Beckman Al. Effect of intrahypothalamic norepinephrine on thermoregulatory responses in the rat. Am J Physiol. 1970;218(8):1596-1604.

43. Freedman RR. Physiology of hot flashes. Am J Hum Biol. 2001;13 (4):453-464.

44. Freedman RR, Woodward S, Sabharwal SC. Alfa2 -adrenergic mechanism in menopausal hot flushes. Obstet Gynecol. 1990;76(4):573-578.

45. Etgen AM, Ansono ff MA, Busada A. Mechanisms of ovarian steroid regulation of norepinephrine receptor-mediated signal transduction in the hypothalamus: implications for female reproductive physiology. Horm Behav. 2001;40:169-177.

46. Rossouw JE, Anderson GL, Prentice RL, LaCroix AZ, Kooperberg C, Stefanick ML, Jackson RD, Beresford SA, Howard BV, Johnson KC, Kotchen JM, Ockene J; Writing Group for the Women's Health Initiative Investigators. Risks and benefits of estrogen plus progestin in healthy postmenopausal women: principal results from the Women's Health Initiative randomized controlled Trial. JAMA. 2002(2);288:321-333. 
47. Freedman RR Krell W. Reduced thermorregulatory null zone in postmenopausal women with hot flashes. Am J Obstet Gynecol. 1999;18(1):66-70.

48. Stearns V, Johnson MD, Rae JM, et al. Paroxetine controlled release in the treatment of menopausal hot flashes: a randomized controlled trial. JAMA. 2003;289 (21):2827-2834.

49. Freedman RR, Dinsay R. Clonidine raises the sweating threshold in symptomatic but not in asymptomatic postmenopausal women. Fertil Steril. 2000;74(1):20-23.

50. Ball P, Gelbke HP, Knuppen Rl. The excretion of 2-hydroxyes trone during the menstrual cycle. J Clin Endocrinol Metab. 1975;40 (3):406-409.

51. Paul SM, Axelrod J. Catechol estrogen - presence in brain and endocrine tissues. Science, 1977;12:197(4304) : 657-659.

52. Stubbs WA, Delitala G, Jones A, et all. Hormonal and metabolic responses to an enkephalin analogue in normal man. Lancet. 1978; 2(8102):1225-1227.

53. Lightman SL, Jacobs HS, Maguire AK, McGarrick G, Jeffcoate SL. Climateric flushing. Clinical and endocrine response to infusion of naloxone. Br J Obstet Gynaecol. 1981;88 (9):919924.

54. Tulandi T, Kinch RA, Guyda H, Maiolo LM, Lal S. Effect of naloxone on menopausal flushes, skin temperature, and luteinizing hormone secretion. Am J Obstet Gynecol. 1985; 151(2):277280 .

55. Shanafelt TD, Barton DL, Adjei AA, Loprinzi CL. Pathophysiology and treatment of hot flashes. Mayo Clin Proc. 2002;77 (11):1207-1218.

56. Curcio JJ, Kim LS, Wollner D, Pockaj BA. The potential role of 5-Hydroxytryptophan for hot flash reduction: A hypothesis. Altern Med Rec. 2005;10(3): 216-221.

57. Dyer RG, Grossman R. Opioid modulation of the response of preoptic neurones to stimulation of the ventral noradrenergic tract in female rats. J Physiol, 1988;20(6):743-749.
58. Fishman J, Norton BL, Hahn EF. Opiate regulation of estradiol2-hydroxylase in the brain of female rats: Mechanism for control of pituitary hormone secretion. Proc Natl Acad Sci USA. 1980;77(3):2574-2576.

59. Loprinzi CL, Michalak JC, Quella SK, O’Fallon JR, Hatfield AK, Nelimark RA, Dose AM, Fischer T, Johnson C, Klatt NE, et al. Megestrol acetate for the prevention of hot flushes. N Engl J Med.1994;331(16): 347-352.

60. Beral V. Million Women Study Collaborators. Breast cancer, hormone replacement therapy in the million women study. Lancet. 2003;362(9282):419-422.

61. Savage MV, Brengelman GL. Control of skin blood flow in the neutral zone of human body temperature regulation. J Appl Physiol. 1996;80(4):1249-1257.

62. Sharpley AL, Cowen PJ. Effect of pharmacologic treatments on the sleep of depressed patients. Biol Psychiatry. 1995;37(2):85-98.

63. Berendsen $\mathrm{HH}$. The role of serotonine in hot flushes. Matiritas. 2000;36(3):155-164.

64. Freedman RR. Pathophysiology and treatment of menopausal hot flashes. Semin Reprod Med. 2005;23(3):117-125.

65. Loprinzi CL, Levitt R, Barton D, Sloan JA, Dakhil SR, Nikcevich DA, Bearden JD 3rd, Mailliard JA, Tschetter LK, Fitch TR, Kugler JW. Phase III comparison of depomedroxyprogesterone acetate to venlafaxine for managing hot flashes: North Central Cancer Treatment Group Trial N99C7. J Clin Oncol. 2006;24 (9): 1409-1414.

Correspondencia autor: Dr. Santiago Vilar González Servicio de Radioterapia. Complejo Hospitalario Univ. de Albacete Hnos. Falco, s/n - 02006 Albacete. Tel.: 0034967597523 E-mail autor: santiagov06@gmail.com Información artículo: Original - Cáncer de próstata Trabajo recibido: octubre 2008

Trabajo aceptado: noviembre 2008 